Documentation et bibliothèques

DOCUMENTATION BIBLIOTHEQUES

\title{
Le droit de prêt public au Canada
}

Public lending right in Canada

El derecho de préstamo público en Canadá

\section{Jules Larivière}

Volume 37, numéro 2, avril-juin 1991

URI : https://id.erudit.org/iderudit/1028446ar

DOI : https://doi.org/10.7202/1028446ar

Aller au sommaire du numéro

\section{Éditeur(s)}

Association pour l'avancement des sciences et des techniques de la documentation (ASTED)

ISSN

0315-2340 (imprimé)

2291-8949 (numérique)

Découvrir la revue

Citer cet article

Larivière, J. (1991). Le droit de prêt public au Canada. Documentation et bibliothèques, 37(2), 53-58. https://doi.org/10.7202/1028446ar
Résumé de l'article

Le droit de prêt public vise à offrir une compensation financière aux écrivains pour la disponibilité gratuite de leurs oeuvres en bibliothèques. Après avoir situé le débat sur la question, l'auteur présente un historique du droit de prêt public. Il décrit ensuite en détail le programme canadien mis en place en 1987.
Tous droits réservés (C) Association pour l'avancement des sciences et des techniques de la documentation (ASTED), 1991
Ce document est protégé par la loi sur le droit d'auteur. L'utilisation des services d'Érudit (y compris la reproduction) est assujettie à sa politique d'utilisation que vous pouvez consulter en ligne.

https://apropos.erudit.org/fr/usagers/politique-dutilisation/ 


\title{
Le droit de prêt public au Canada
}

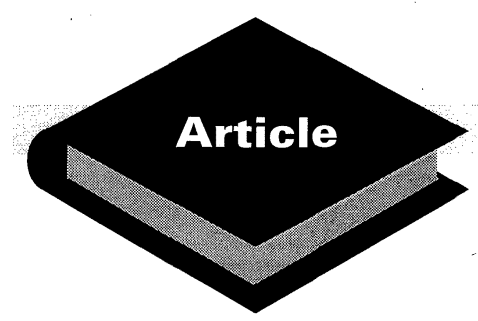

\author{
Jules Larivière \\ Bibliothèque de Droit \\ Université d'Ottawa
}

Le droit de prêt public vise à offrir une compensation financière aux écrivains pour la disponibilité gratuite de leurs oeuvres en bibliothèques. Après avoir situé le débat sur la question, l'auteur présente un historique du droit de prêt public. Il décrit ensuite en détail le programme canadien mis en place en 1987.

\section{Public lending right in Canada}

Public lending right compensates authors in return for the free access of their works in libraries. The author focuses on the parameters of the issue and then describes its evolution. A lengthy description of the Canadian programme, set up in 1987, follows.

\section{El derecho de préstamo público en Canadá}

El derecho de préstamo público aspira a ofrecer una compensación financiera a los escritores para la disponibilidad gratuita de sus obras en las bibliotecas. Después de situar el debate sobre la cuestión, el autor presenta un resumen histórico del derecho de préstamo público. Por fin, describe detalladamente el programa canadiense establecido en 1987.
En avril 1986, le Canada devient le onzième pays à offrir une compensation financière à ses écrivains pour la présence de leurs livres dans les bibliothèques. En reconnaissant aux auteurs canadiens le droit à une compensation monétaire pour la disponibilité gratuite de leurs oeuvres en bibliothèques, le gouvernement canadien répond à l'argumentation des organismes d'écrivains qui, depuis quarante ans, réclament un tel paiement.

Avant le Canada, le Danemark en 1946, la Norvège en 1947, la Suède en 1954, la Finlande en 1961, I'Islande en 1968, les Pays-Bas en 1971, I'Allemagne de l'Ouest en 1972, la NouvelleZélande en 1973, I'Australie en 1976 et la Grande-Bretagne en 1979 avaient déjà implanté des programmes de compensation pour le prêt public en bibliothèques. Depuis, Israël a également mis sur pied en 1988 un programme de paiement aux écrivains pour le prêt public.

La prétention des auteurs à disposer d'un droit à une rémunération chaque fois que l'une de leurs oeuvres est prêtée dans une bibliothèque ne fait pas nécessairement l'unanimité. En effet, à cette prétention, on allègue que l'écrivain ne peut prétendre à un droit de contrôle sur la circulation des exemplaires de ses livres pour lesquels il a déjà été compensé lors de
I'achat des exemplaires par les bibliothèques qui prêtent ces livres gratuitement.

\section{Le débat sur la question du droit de prêt public}

C'est en invoquant un manque à gagner chaque fois qu'un livre est mis gratuitement à la disposition des clientèles dans les bibliothèques que les organisations d'écrivains mènent leur bataille visant à faire reconnaître un droit à une compensation monétaire pour une telle utilisation de leurs livres. Dans tous les pays où se tient le débat sur le droit de prêt public, la question de la rémunération des auteurs pour l'utilisation publique de leurs livres dans les bibliothèques suscite de vives discussions ${ }^{1}$. Malheureusement, le ton passionné d'un débat parfois empreint de vives émotions empêche souvent une analyse objective et rationnelle de la question.

Alors que d'un côté, certains écrivains considèrent le droit de prêt public comme une simple question de justice sociale et le voient comme un encouragement normal à la création littéraire, d'autres prétendent que les écrivains eux-mêmes ignorent ce principe de justice en réclamant, dans la plupart des systèmes en place, des exceptions qui, selon eux, ne devraient pas être compensées ${ }^{2}$.
De leur côté, les bibliothécaires soutiennent qu'il est faux de prétendre qu'une personne qui n'aurait pas un accès facile et gratuit à un livre dans une bibliothèque achèterait nécessairement I'ouvrage. Pour plusieurs, il s'agit finalement d'une autre façon de subventionner les écrivains dont les oeuvres sont peu lues, puisque la plupart des écrivains s'opposent au système reposant sur les données statistiques de prêt, y préférant celui basé sur les données de fonds de collections.

Et pendant ce temps, certains juristes s'interrogent à savoir si :

les droits pécuniaires traditionnels reconnus aux auteurs, droit de reproduction et droit de représentation publique, constituent la limite extrême de la protection des créateurs ou s'il ne convient pas de réserver aussi à l'auteur la haute main sur l'exploitation des exemplaires de son oeuvre, même après $q u$ 'il en ait autorisé la fabrication et la vente ${ }^{3}$.

1. Thomas Stave, "Public Lending Right: a History of the Idea ", Library Trends, vol. 29, no. 4 (Spring 1981), 570.

2. Ibid., 571.

3. F. Pollaud-Dulian, Le droit de destination; le sort des exemplaires en droit d'auteur, Paris Librairie générale de droit et de jurisprudence, 1989 , p. 17. 
D'autres juristes, par ailleurs, soutiennent qu'en vertu de la dóctrine de l'épuisement, un auteur ne saurait prétendre à un contrôle sur la distribution et la circulation de son oeuvre, son droit s'épuisant une fois qu'il a autorisé une première vente des exemplaires ${ }^{4}$.

Le principal problème soulevé par le droit de prêt public est évidemment la gratuité du prêt dans les bibliothèques. En effet, la plupart des intervenants s'entendent pour reconnaître aux créateurs une certaine prétention lucrative à la location des oeuvres; cependant, la gratuité du prêt en bibliothèque enlèverait, selon certains, toute valeur économique négociable. Mais pour d'autres, situer le débat du droit de prêt public au niveau de celui de l'accessibilité à l'information documentaire risque de forcer l'écrivain à renoncer à son droit pécuniaire pour le prêt de ses livres. L'intérêt général ou collectif prétendant un accès égal pour tous à l'éducation et à la culture peut-il justifier qu'un auteur doive renoncer à sa propriété et aux droits qui en découlent, y compris celui à une juste rémunération de son travail créateur? Le débat est toujours ouvert sur cette question: ou bien on reconnaît ce droit et on intègre le droit de prêt public dans les diverses législations nationales sur le droit d'auteur en s'assurant que la loi ne souffre $d^{\prime}$ aucune discrimination entre les auteurs, y compris la réciprocité de traitement des auteurs étrangers en vertu de la Convention de Berne, ou bien on compense les auteurs de la perte du droit de contrôle sur l'utilisation gratuite par l'entremise de programmes gouvernementaux qui sont alors régis par une simple réglementation administrative, ou, finalement, on retient l'argument que le prêt public gratuit ne cause pas de préjudice financier à l'auteur, puisque l'emprunteur n'emprunte pas nécessairement au lieu d'acheter. À l'exception de l'Allemagne, aucun des pays accordant une compensation aux auteurs pour le prêt public ne l'a fait dans le cadre de sa législation sur le droit d'auteur ${ }^{5}$.

Certains cependant l'ont fait à l'intérieur de leurs lois sur les bibliothèques publiques ${ }^{6}$.

\section{L'histoire du droit de prêt public}

L'idée du droit de prêt public est relativement jeune et représente un nouveau genre de droit. L'évolution historique et le contexte politico-social dans lesquels elle s'est développée sont assez importants pour qu'on s'y attarde un moment.

C'est dans les pays scandinaves que commence, au début du vingtième siècle, à être soulevée la possibilité de payer une redevance aux auteurs pour le prêt de leurs livres par les bibliothèques. En effet, la première proposition sérieuse dans ce sens est faite en 1918 au Danemark. On recommande alors l'imposition d'une taxe sur chaque prêt d'un livre écrit par un écrivain danois. Deux raisons principales expliquent le développement de cette idée à l'intérieur des pays scandinaves: d'abord, le contexte socialdémocrate de ces pays nordiques où on place le bien-être de toutes les classes de la population au même niveau, et ensuite le marché relativement restreint des écrivains qui oblige à limiter les éditions ${ }^{7}$. L'idée de la taxe est cependant vite rejetée parce qu'elle vient en contradiction directe avec le principe inattaquable de l'accès gratuit aux bibliothèques publiques. II est intéressant de noter ici que le débat entourant la question n'est pas seulement théorique. En 1929, un auteur danois, Peter Freuchen, publie un ouvrage qui porte une mention interdisant le prêt du livre sans la permission de l'auteur. II y précise que cette permission sera accordée moyennant une compensation. La Cour est appelée à se prononcer et reconnaît le droit à l'auteur d'imposer une telle restriction. Fortes de cette reconnaissance, les organisations d'écrivains des divers pays nordiques entreprennent des négociations sérieuses avec leurs divers gouvernements. C'est finalement en 1946 que le Parlement danois vote un amendement à la Loi sur les bibliothèques publiques, lequel octroie aux écrivains danois une compensation financière pour la présence de leurs livres dans les bibliothèques.

L'année suivante, c'est au tour de la Norvège de reconnaître légalement une compensation aux écrivains. Dans ce cas cependant, il s'agit de la création d'un fonds qui doit servir à aider les jeunes auteurs et les écrivains éprouvant des difficultés financières. C'est cependant en 1954, en Suède, qu'on met sur pied le premier véritable système de droit de prêt public, c'està-dire un système de paiement à partir des statistiques de prêts dans les bibliothèques publiques. Puis, en 1961, la Finlande implante un système similaire à celui de la Norvège. Le dernier pays scandinave à reconnaître un droit de compensation est I'Islande en 1968. De ces premiers systèmes de droit de prêt public, on peut retenir deux points importants : d'abord, tous ont une base législative permettant une certaine sécurité de permanence aux programmes et enfin, aucun n'est subventionné à partir des budgets de bibliothèques, les fonds provenant dans les cinq cas directement des gouvernements.

En 1971, les Pays-Bas innovent en développant un programme administratif qui repose sur la compensation en vertu des budgets d'acquisitions des livres des écrivains littéraires du pays.

Mais c'est véritablement en 1972 que le droit de prêt public devient un concept juridique reconnu alors que I'Allemagne incorpore le droit de prêt public à sa loi sur le droit d'auteur. En reconnaissant le droit de prêt public en tant que composante du droit de distribution, le législateur allemand donne à l'idée de compensation pour le prêt de livres en bibliothèque une base juridique ${ }^{8}$.

Poursuivant lentement mais sûrement son développement à travers divers pays, c'est en 1973 que le droit de prêt public est reconnu en NouvelleZélande dans le cadre de la création d'un "fonds d'auteurs " à partir duquel on compenserait les écrivains dont les livres se trouvent dans les collections des bibliothèques publiques, universitaires et collégiales.

4. Ibid., p. 347.

5. H. Cohen Jehoram, ed. Public Lending Right: Reports of an ALAI Symposium and Additional Materials, Amsterdam, Kluwer, 1983 p. , 180-181

6. P. Kirkegaard, Author's Lending Right in Scandinavia, Proceedings, Canadian Library Association, Twenty-Seventh Annual Conference, Regina, Saskatchewan, 10-16 June 1972, Ottawa, Canadian Library Association, 1972, p. 84.

7. Ibid., p. 83.

8. G. McFarlane, "Le droit de prêt public - Une comparaison de diverses conceptions nationales ", Le Droit d'auteur (novembre 1980), 278. 
En 1976, I'Australie se joint au mouvement de reconnaissance du droit de prêt public, alors qu'un comité du droit de prêt public est établi à I'intérieur du Conseil des arts d'Australie. Basé sur le paiement aux auteurs australiens à partir d'un échantillonnage d'un certain nombre de bibliothèques, il s'agit d'un système qui tient compte des fonds de collection seulement.

Au Royaume-Uni, la mise en place en 1979 d'un programme gouvernemental de compensation pour le prêt des livres en bibliothèques est aussi le résultat d'une longue bataille menée par les écrivains. Dès 1951, des écrivains suggèrent qu'on paie une redevance chaque fois qu'on emprunte un livre dans les bibliothèques. Mais

au fil des ans, les oppositions ont été nombreuses et, bien que le Parlement eût été saisi à diverses reprises de projets de loi tendant à introduire ce droit dans la législation du Royaume-Uni, ces textes avaient toujours été repoussés ou retirés, parfois au milieu de vives controverses $^{9}$.

La base du système britannique est le prêt des livres à l'intérieur d'un échantillonnage de bibliothèques identifiées par l'organisme administrant le programme.

\section{Le programme canadien de droit de prêt public}

C'est donc en mars 1987 que la Commission canadienne de droit de prêt public met à la poste ses premiers chèques destinés à offrir une compensation aux auteurs canadiens dont les livres sont prêtés par les bibliothèques canadiennes. La cérémonie protocolaire du 17 mars 1987, lorsque la ministre des Communications, Flora MacDonald, remet un chèque à un écrivain, marque l'aboutissement d'une longue bataille pour les organismes nationaux d'écrivains. Pour les organismes de bibliothécaires, elle signifie la fin d'une situation inconfortable, soutenant d'un côté les écrivains pour une amélioration de leur situation financière, mais s'opposant d'un autre côté à la prétention que le prêt gratuit affecte les revenus potentiels des écrivains ${ }^{10}$. De plus, les bibliothécaires canadiens ont toujours craint qu'un programme de compensation financière pour le prêt des livres vienne gruger des budgets déjà restreints et ajoute une surcharge administrative à un personnel déjà insuffisant. Finalement, les deux associations nationales de bibliothécaires (la Canadian Library Association et I'Association pour l'avancement des sciences et des techniques de la documentation) soutenaient qu'un véritable programme de droit de prêt public, c'est-à-dire reposant sur les statistiques véritables de prêts, bénéficierait surtout aux écrivains les plus populaires et par conséquent le moins dans le besoin ${ }^{11}$.

Au niveau gouvernemental, c'est le Conseil des arts du Canada qui pilote le dossier du droit de prêt public. Dès 1974, sa Commission consultative des arts recommande qu'on rédige, après consultation des principaux intervenants (écrivains, bibliothécaires et éditeurs), un document de travail sur la question. Conscient de la polémique entre auteurs et bibliothécaires sur le sujet, le Conseil des arts attend 1976 avant de mettre sur pied un comité d'étude. Fort de l'appui des deux associations nationales de bibliothécaires qui ont toutes deux voté cette année-là une résolution qui "encourageait le gouvernement fédéral à créer et financer un système de compensation financière pour les auteurs " 12 , le Conseil donne à son comité d'étude le "mandat de concevoir un système adapté au Canada et de déterminer les fonds nécessaires à sa mise en pratique "13. Le 6 octobre 1981, le Conseil des arts soumet au ministre des Communications une proposition détaillée en vue de l'établissement d'un programme de droit de prêt public au Canada. II faut alors attendre jusqu'en 1984 pour voir ce dossier progresser à l'intérieur de la bureaucratie gouvernementale. En effet, l'arrivée au pouvoir du Parti progressiste-conservateur, et plus particulièrement la nomination de Marcel Masse à titre de ministre des Communications, permettent un déblocage, compte tenu de la volonté politique de reconnaître une forme de compensation.

En avril 1986, le ministère des Communications, sous l'égide du Conseil des arts du Canada, affecte une somme de trois millions de dollars et, en septembre de la même année, crée une Commission du droit de prêt public. Formée de 24 membres représentant toutes les associations natio- nales d'écrivains, de bibliothécaires et d'éditeurs, la Commission reçoit le mandat "de payer les auteurs canadiens dont les livres étaient utilisés dans les bibliothèques " ${ }^{14}$. Sept mois plus tard, 4377 écrivains canadiens se partageaient 2712094 \$ en compensation de 15654 titres répertoriés dans les bibliothèques sélectionnées.

Le système canadien du droit de prêt public est basé sur un paiement annuel aux auteurs canadiens pour chaque titre admissible catalogué et disponible dans une sélection de bibliothèques publiques et universitaires. Sous réserve de certaines conditions d'admissibilité, les auteurs doivent inscrire leurs oeuvres auprès de la Commission qui établit une liste des titres admissibles. Cette liste est ensuite comparée aux collections de 15 bibliothèques représentatives (10 anglophones et 5 francophones). Donc, plus il y a de bibliothèques qui détiennent un même titre, plus le paiement augmente, un auteur ne pouvant recevoir cependant plus de $4000 \$$ par année du programme. Dans l'établissement de l'échelle de paiement par titre retrouvé, on prend en considération la rentabilité potentielle à l'intérieur des bibliothèques anglophones et des bibliothèques francophones, compte tenu du nombre de bibliothèques répertoriées dans les deux cas. Ainsi, un titre dans une bibliothèque anglophone est payé $40 \$$, tandis qu'on paie $80 \$$ pour un titre dans une bibliothèque francophone; de la même façon, on paie $400 \$$ pour un titre retrouvé dans les dix (10) bibliothèques anglophones et $400 \$$ pour un titre retrouvé dans les cinq (5) bibliothèques francophones. Lors de l'échantillonnage préliminaire, on a également réalisé que les livres pour enfants allaient être handicapés par leur performance dans les bibliothèques universitaires. On tient donc

9. G. McFarlane, "La loi de 1979 du RoyaumeUni. sur le droit de prêt public ", Le Droit d'auteur (décembre 1979), 325.

10. A.B. Piternick, "Library Royalties in Canada ", LIBRI, vol. 28, no. 3 (September 1978), 246.

11. Ibid., 247.

12. Conseil des arts du Canada. Proposition concernant l'établissement d'un système de paiement aux auteurs canadiens pour l'utilisation publique de leurs livres dans les bibliothèques du Canada, Ottawa, 1981, p. 1.

13. Ibid.

14. Commission du droit de prêt public, Rapport annuel, 1986/1987, p. 1. 


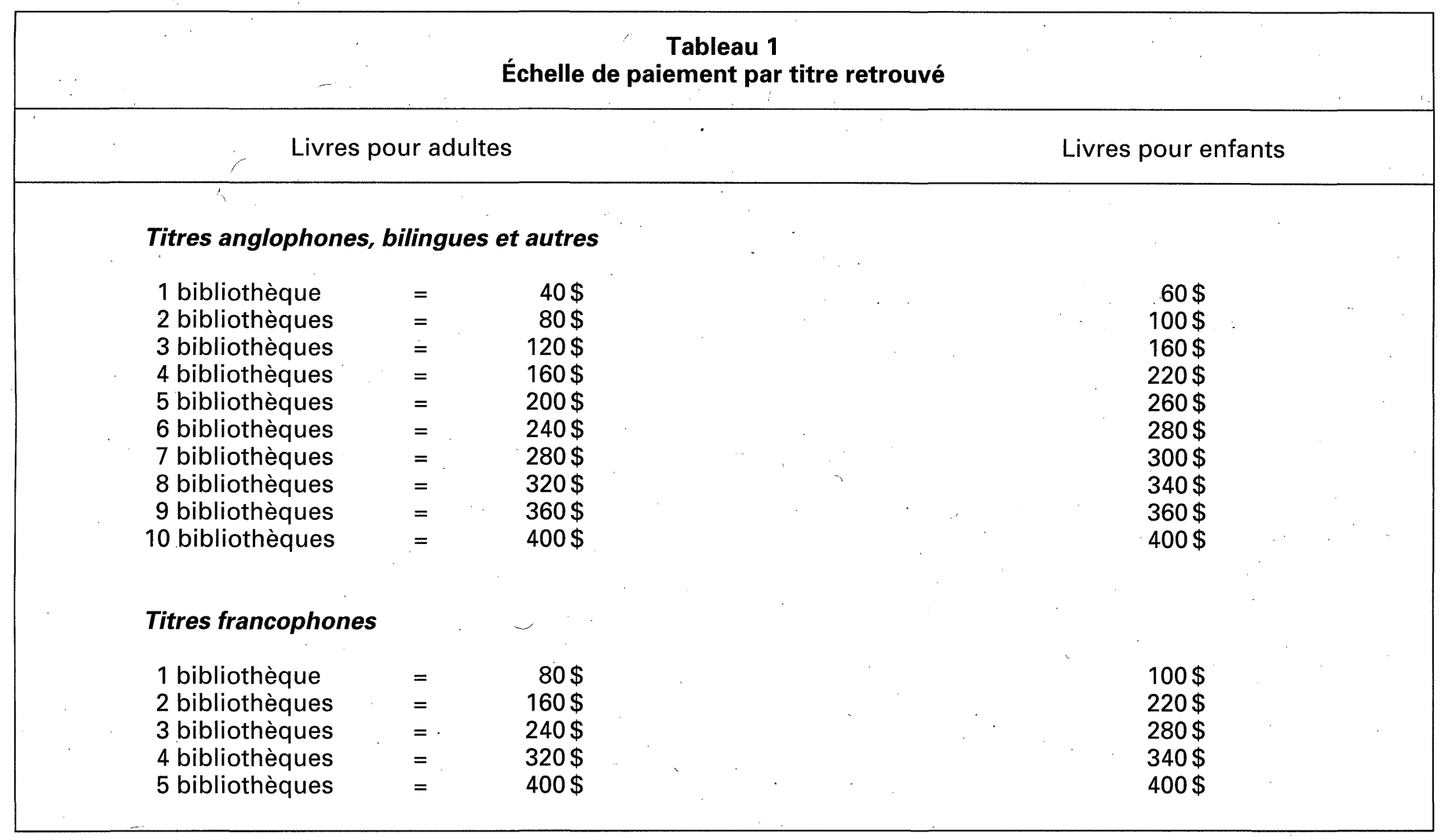

compte de cette situation dans l'échelle de paiement: un titre pour enfants dans une bibliothèque anglophone reçoit $60 \$$ et un titre pour enfants dans une bibliothèque francophone reçoit $100 \$$. Le tableau 1 donne plus de détails sur les montants d'argent payés aux auteurs selon le nombre de fois que leurs titres sont retrouvés dans les différentes bibliothèques. II faut se rappeler que les titres en anglais, bilingues et dans les -autres langues sont vérifiés uniquement dans les bibliothèques anglophones, alors que les titres en langue française sont vérifiés uniquement dans les bibliothèques francophones.

L'auteur retire $100 \%$ du paiement s'il n'y a pas d'autres collaborateurs. En effet, le programme canadien offre aussi une compensation aux traducteurs, aux illustrateurs, aux directeurs de rédaction et aux collaborateurs d'une anthologie.

Pour être admissible à un paiement de la part de la Commission canadienne de droit de prêt public, un auteur et ses collaborateurs doivent répondre aux critères suivants:
- être citoyen canadien (vivant au Canada ou à l'étranger) ou résident permanent du Canada ;

- voir son nom apparaître sur la page de titre de l'ouvrage inscrit au programme ;

- inscrire chaque titre en accompagnant le formulaire d'une photocopie de la page de titre et de la table des matières;

- ne pas avoir écrit le livre pour son employeur dans le cadre de son emploi ;

- I'ouvrage inscrit doit comprendre au moins 48 pages et dans le cas d'un livre pour enfants, au moins 24 pages.

Même si le programme canadien se veut aussi large que possible, il comprend toutefois un certain nombre d'exceptions; ainsi les catégories suivantes d'ouvrages ne sont pas admissibles à un paiement:

- les journaux, revues ou périodiques ;
- les manuels scolaires et les ouvrages principalement destinés à l'enseignement;

- les répertoires, index, compilations, bibliographies, dictionnaires, atlas et encyclopédies;

- les généalogies;

- les rapports d'étude, d'enquête, d'analyse ou d'évaluation préparés pour ou publiés par un organisme, société ou établissement gouvernemental ou para-gouvernemental;

- les manuels d'instruction portant sur le fonctionnement d'appareils ou sur les techniques de jeux;

- les ouvrages pratiques, comme les livres de recettes culinaires, les guides touristiques et autres;

- les livres à colorier ;

- les partitions musicales;

- les thèses non publiées ;

- les manuscrits; 


\begin{tabular}{|lrrrr|}
\hline & $\begin{array}{c}\text { Tableau 2 } \\
\text { Bilan }\end{array}$ & & & \\
& $1986 / 87$ & $1987 / 88$ & $1988 / 89$ & $1989 / 90$ \\
\hline Nombre d'auteurs inscrits & 4553 & 5635 & 6713 & 7153 \\
Nombre d'auteurs recevant un paiement & 4377 & 5200 & 5718 & 6405 \\
Nombre de titres inscrits & 16557 & 21721 & 28259 & 31375 \\
Nombre de titres admissibles & 15654 & 18850 & 23689 & 24891 \\
Somme versée & $2712094 \$$ & $3484988 \$$ & $3970947 \$$ & $4667214 \$$ \\
\hline
\end{tabular}

\begin{tabular}{|lrrrr|}
\hline \multicolumn{4}{c}{ Tableau 3 } & \\
& Analyse des inscriptions de titres par langue du texte & & \\
\hline & $1986 / 87$ & $1987 / 88$ & $1988 / 89$ & $1989 / 90$ \\
\hline Anglais & 10838 & 14258 & 17948 & 19859 \\
Français & 5205 & 6769 & 9271 & 10345 \\
Bilingues & 185 & 207 & 278 & 290 \\
Autres langues & 329 & 487 & 762 & 881 \\
Total & 16557 & 21721 & 28259 & 31375 \\
\hline
\end{tabular}

- les catalogues d'exposition;

- les actes de colloques, séminaires ou congrès ;

- les agendas et les calendriers.

La question des critères d'admissibilité est un des principaux problèmes qui confrontent actuellement la Commission. Les pressions sont fortes pour rendre le programme universel, tant au niveau du gouvernement que de certains groupes d'auteurs. C'est d'ailleurs dans ce contexte que la Commission a créé un groupe de travail en 1989 pour étudier la pertinence des critères actuellement en place ${ }^{15}$.

Le 31 mars 1990 prenait fin la quatrième année du programme canadien de compensation aux auteurs canadiens pour la disponibilité gratuite de leurs oeuvres dans les bibliothèques. Dans l'ensemble, il s'agit d'un programme maintenant bien rodé dont l'administration est finalement simple et efficace. De plus, le gouvernement a décidé d'ajouter des fonds supplémentaires de façon à maintenir l'échelle de paiement, compte tenu du nombre grandissant d'auteurs s'inscrivant et de titres admissibles, comme I'indique le tableau 2.

L'analyse des données státistiques pour les quatre premières années du programme révèle que le rapport entre les titres inscrits en langue anglaise et ceux de langue françaìse est à peu de chose près de 2 pour 1 . Le tableau 3 donne une analyse des inscriptions.

Quant à la répartition des paiements par genres d'ouvrages, les statistiques du tableau 4 démontrent clairementque la catégorie des ouvrages dits non littéraires domine et que, s'il fallait restreindre le programme aux auteurs littéraires seulement, les paiements seraient plus intéressants pour cette catégorie qui en a peut-être un plus grand besoin. II s'agit là d'un débat actuellement en cours à l'intérieur de la Commission.

Finalement, le tableau 5 donne un aperçu de la moyenne du taux de présence dans les bibliothèques sélectionnées et des titres répertoriés au cours des quatre premières années du programme. Il est intéressant de noter que, dès sa création, la Commission canadienne de droit de prêt public a décidé de ne pas faire connaître publiquement le nom des bibliothèques inventoriées. Elle rencontre ainsi

15. Commission du droit de prêt public, Rapport annuel, 1988/1989, p. 5 
Tableau 4

Répartition des paiements par genre de livres

\begin{tabular}{|lrrrr|}
\hline & $1986 / 87$ & $1987 / 88$ & $1988 / 89$ & $1989 / 90$ \\
\hline Livres pour enfants & 1591 & 2041 & 2411 & 2808 \\
Théâtre & 450 & 513 & 607 & 664 \\
Romans/nouvelles & 2800 & 3488 & 4080 & 4631 \\
Poésie & 2221 & 2732 & 3021 & 3309 \\
Non littéraires & 8592 & 9616 & 9966 & 12004 \\
\hline
\end{tabular}

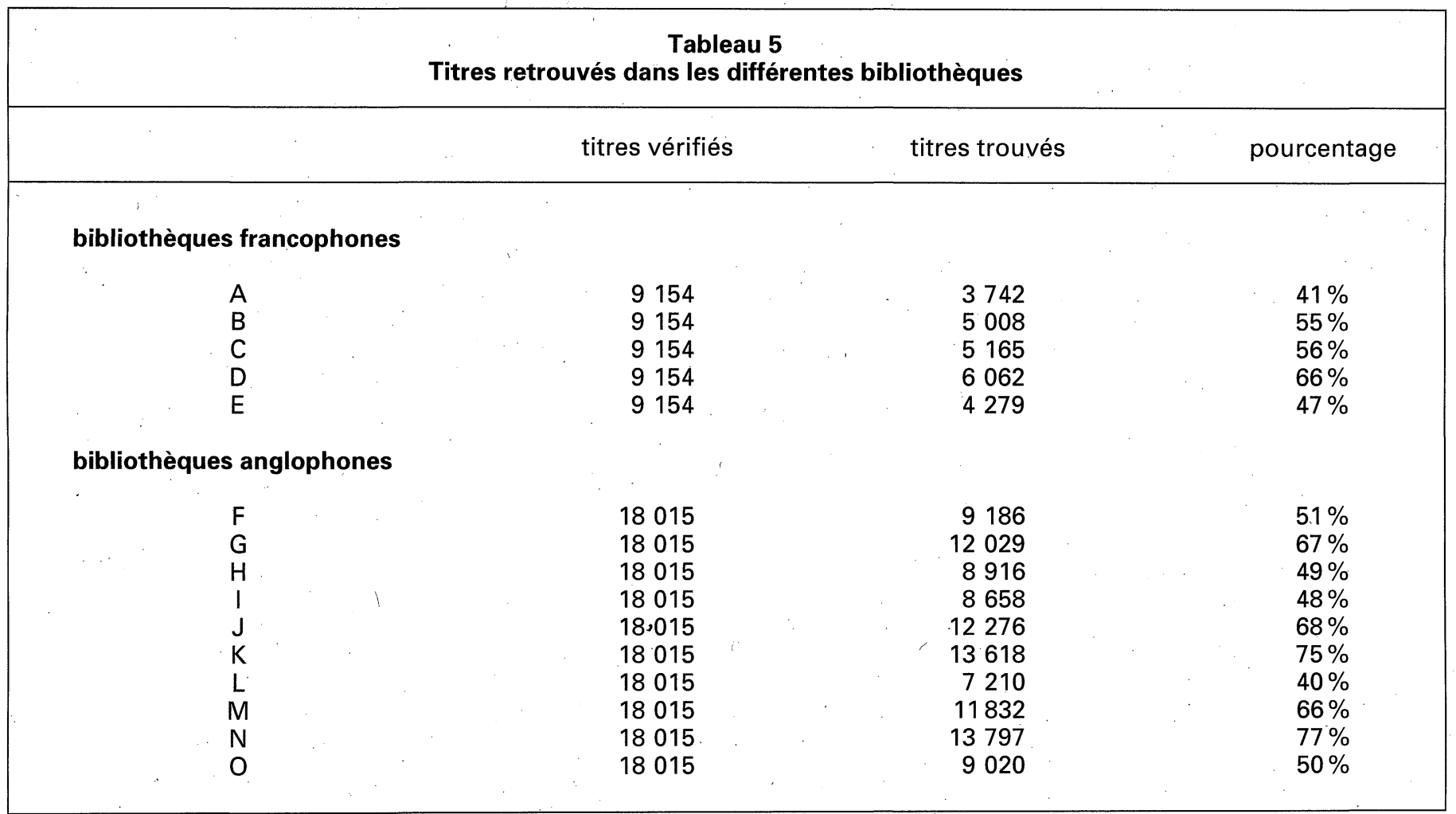

une demande des bibliothécaires qui ne voulaient pas subir de pressions de la part des auteurs et voulaient éviter qu'on se serve des données pour " "évaluer" la valeur "canadienne" de leurs fonds de collections.

\section{Conclusion}

L'idée d'offrir une compensation aux auteurs pour le prêt gratuit de leurs livres dans les bibliothèques surprend toujours, tellement on est habitué à la situation. Mais la réflexion force à reconnaître qu'il y a effectivement une certaine injustice à ne pas reconnaître à l'auteur un droit à une compensation. La question est maintenant de savoir comment on peut reconnaître ce droit et jusqu'à quel degré. Le débat sur le principe même de ce droit, ainsi que sur les objectifs à atteindre en le reconnaissant, reste ouvert. Entre temps, divers pays ont des mécanismes qui semblent fonctionner et satisfaire les auteurs alors que d'au- tres, comme la Belgique et les ÉtatsUnis, considèrent le droit de prêt public comme souhaitable et réalisable dans un avenir rapproché.

Au Canada, le programme de droit de prêt public est simple et modeste, mais il progresse sûrement. De plus en plus connu, comme en témoignent les statistiques, il repose sur une participation active de tous les intervenants intéressés, c'est-à-dire écrivains, éditeurs et bibliothécaires. 